\title{
A STEREOLOGICAL UBIQUITIFORMAL SOFTENING MODEL FOR CONCRETE
}

\author{
Zhuo-Cheng Ou, Guan-Ying Li, Zhuo-Ping Duan, Feng-Lei Huang \\ State Key Laboratory of Explosion Science and Technology, Beijing Institute of Technology, Beijing, China \\ e-mail address: zcou@bit.edu.cn
}

\begin{abstract}
A stereological ubiquitiformal softening model for describing the softening behavior of concrete under quasi-static uniaxial tensile loadings is presented in this paper. In the model, both the damage evaluation process of fracture cross-sections and their distribution along the specimens axis are taken into account. The numerical results of a certain kind of full grade concrete made of crushed coarse aggregate are found to be in good agreement with the experimental data. Moreover, an experiental relation between the lower bound to the scale invariance of concrete and its tensile strength is also obtained by data fitting of the experimental data, which provides an effective approach to determine the lower bound to scale invariance of concrete.
\end{abstract}

Keywords: ubiquitiform, fractal, concrete, softening curve

\section{Introduction}

Fractals have been widely used as a nonlinear mathematical tool to describe mechanical behavior of heterogeneous materials such as concrete since the pioneer work of Mandelbrot (1982), Mandelbrot et al. (1984). It has been found that the internal structure of concrete appears quite a well approximate self-similarity in many aspects over certain ranges of scale. For example, it has been verified experimentally that the fracture surface of concrete can be described by fractals (Saouma and Barton, 1994; Charkaluk et al., 1998). Stroeven has shown that for almost all the aggregate grading in concrete, the distribution of the aggregate particles in various diameters appears the self-similarity feature (Stroeven, 1973, 2000). Moreover, fractals have also been widely used to describe the fracture behavior of concrete (Borodich, 1997; Carpinteri et al., 2002; Khezrzadeh and Mofid, 2006). However, there are still many intrinsic difficulties in fractal applications, especially in the case when the measure of a real geometrical or physical object must be taken into account because kinds of density of fractal parameters defined on the unit fractal measure are not only lacking unambiguous physical meanings but also very difficult to be determined in practice. Recently, Ou et al. (2014) demonstrated that such a difficulty was caused by contradiction between the integral dimensional immeasurability of a fractal and the integral dimensional characteristic of a real physical or geometrical object in nature, and proposed a new concept of ubiquitiform. According to Ou et al. (2014), a ubiquitiform is defined as a finite order self-similar (or self-affine) physical configuration constructed usually by a finite iterative procedure. It has been shown that a ubiquitiform has a finite integral dimensional measure and must be of integral dimension in Euclidean space, whereas the Hausdorff dimension of a fractal is usually not integral. The Hausdorff dimension of the initial element of a fractal changes abruptly at the point of infinite iteration, which results in divergence of the integral dimensional measure of the fractal and makes the fractal approximation of a real geometrical or physical object to a ubiquitiform unreasonable.

One important phenomenon in tensile failure of concrete is softening, and the most widely used theory is the so-called cohesive crack model (Barenblatt, 1959, 1962). Over the past decades, 
several softening curves have been proposed, such as the linear curve (Hillerborg et al., 1976), the bilinear curve (Petersson, 1981), the nonlinear curve (Reinhardt et al., 1986) and the power-law curve (Gopalaratnam and Shah, 1985; Karihaloo, 1995). Recently, Khezrzadeh and Mofid (2006) proposed a quasi-fractal softening curve based on fractal concepts, in which, however, only the damage evaluation process of the fractured cross-section was considered. On the other hand, as demonstrated by $\mathrm{Ou}$ et al. (2014), a ubiquitiform, rather than a fractal, should be used in describing a real geometrical or physical object in the case of the integral dimensional measure of the object.

Therefore, in this study, based on the concept of the ubiquitiform, a stereological ubiquitiformal softening model for concrete, in which both the damage evaluation process of fractured cross-sections and their distribution along the specimens axis are taken into account, and the calculated results of softening curves of concrete are compared with previous experimental data. Moreover, it is interesting to find that there exists a good correspondence between the lower bound to scale invariance and the tensile strength of concrete, and then an experiential formula for the corresponding relationship is obtained.

\section{Stereological ubiquitiformal softening model}

To describe the damage evaluation process of a concrete specimen, a stereological damage region is assumed in this paper, based on the fracture band theory (Bažant and Oh, 1983). Namely, fracture of a heterogeneous aggregate material such as concrete can be assumed to occur in the form of a blunt smeared crack band. Such a stereological damage region consists of a series of fracture surfaces distributed along the axis of the specimen as a generalized ubiquitiformal Cantor set, and each of the fracture surface will be described by a generalized ubiquitiformal Sierpinski carpet having different complexity. The generalized ubiquitiformal Sierpinski carpet is generated by a series of recursive procedures, i.e. an iteration process from the initial square of unit side length. In each step of the iteration, each remaining square is divided into $p^{2}$ identical smaller squares, and the generalized ubiquitiformal Sierpinski carpet is then obtained by repeatedly removing $q\left(q / p^{2}<1\right)$ small squares from the remaining squares. According to Khezrzadeh and Mofid (2006), the removing area represents the cracked area of the fractured cross-sections. As has been defined by Ou et al. (2014), the complexity $D$ of such a generalized ubiquitiformal Sierpinski carpet is

$$
D=\frac{\ln \left(p^{2}-q\right)}{\ln p}
$$

Therefore, taking different values of $p$ and $q$, the generalized ubiquitiformal Sierpinski carpet can be used to describe a surface with any complexity. The removed area in the $n$-th iteration is

$$
\Delta a_{n}=A_{p} \frac{q}{p^{2}}\left(\frac{p^{2}-q}{p^{2}}\right)^{n-1}
$$

where $A_{p}$ is the nominal area of the generalized ubiquitiformal Sierpinski carpet. The total area of the increased crack surface, when the specimen is failed, is

$$
A_{1}=\sum_{n=1}^{n_{c}} \Delta a_{n}=A_{p}\left[1-\left(\frac{p^{2}-q}{p^{2}}\right)^{n_{c}}\right]=A_{p}\left[1-\left(\frac{1}{p^{n_{c}}}\right)^{2-D}\right]
$$

where $n_{c}$ represents the critical iteration number of the generalized ubiquitiformal Sierpinski carpet when the specimen is failed.

According to the fracture band theory (Bažant and Oh, 1983), in this study, a multiple crack surface hypothesis is proposed in the ubiquitiformal softening model. That is to say, to 
describe the damage evolution of the concrete material, besides the main crack surface, there are still several secondary crack surfaces, each of which is described as the above-mentioned generalized ubiquitiformal Sierpinski carpet with different iteration orders. These crack surfaces are assumed to be distributed along the axis of the specimen as a generalized ubiquitiformal Cantor set (Fig. 1). Hereinafter, we denote these crack surfaces as the $i$-th order crack surfaces $(i=1,2,3, \ldots, m)$, and the first order $(i=1)$ one is the main crack surface. According to the structure of the generalized ubiquitiformal Cantor set, the number of the $i$-th order crack surface is $k_{i}=2^{i-1}$. It is also assumed that the iteration number of the $i$-th order crack surface is one less than that of the $(i-1)$-th order crack surface. Thus, the increase of the $i$-th order crack surface in the $n$-th iteration can be calculated by the equation

$$
\Delta a_{n}^{i}=A_{p} \frac{q}{p^{2}}\left(\frac{p^{2}-q}{p^{2}}\right)^{n-i}
$$

The total increase of the crack surface in the $n$-th iteration is

$$
\Delta a_{n}=\sum_{i=1}^{m} k_{i} \Delta a_{n}^{i}=\sum_{i=1}^{m} 2^{i-1} A_{p} \frac{q}{p^{2}}\left(\frac{p^{2}-q}{p^{2}}\right)^{n-i}=A_{p} \frac{2^{m} q p^{2 m}\left(p^{2}-q\right)^{n-m}-q\left(p^{2}-q\right)^{n}}{p^{2 n}\left(p^{2}+q\right)}
$$

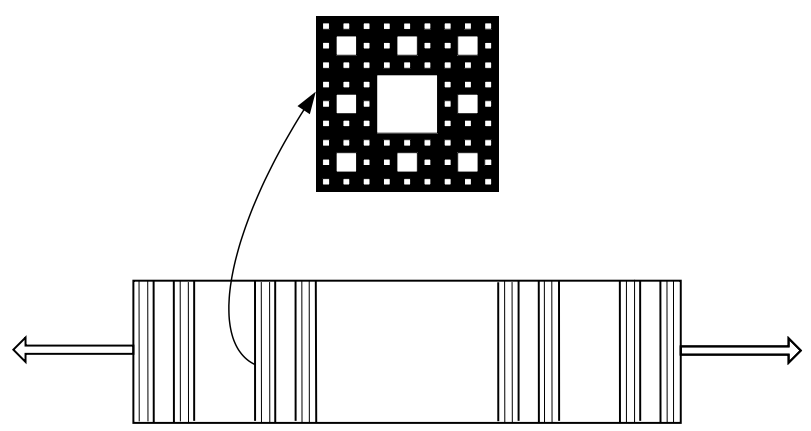

Fig. 1. Stereological ubiquitiformal softening model

According to the iteration law of the generalized ubiquitiformal Sierpinski carpet, the ratio of the area of the $(i+1)$-th crack surface to that of the $i$-th crack surface is

$$
A_{i+1}=\frac{p^{2}-q}{p^{2}} A_{i}
$$

where $i=1,2,3, \ldots, m$, and, from Eq. (2.6), we have

$$
A_{i}=\left(\frac{p^{2}-q}{p^{2}}\right)^{i-1} A_{1}
$$

Then, the total crack surface increased in the fracture process is

$$
A=\sum_{i=1}^{m} k_{i} A_{i}=A_{1} \sum_{i=1}^{m} 2^{i-1}\left(\frac{p^{2}-q}{p^{2}}\right)^{i-1}=A_{1} \frac{p^{2 m}-2^{m}\left(p^{2}-q\right)^{m}}{\left(2 q-p^{2}\right) p^{2 m-2}}
$$

For convenience, here, the homogeneous deformation along the axis of the specimen is assumed, and then the elongations generated in each iteration $\Delta w$ are the same, which can be written as

$$
\Delta w=\frac{w_{c}}{n_{c}}
$$

where $w_{c}$ is the critical elongation of the specimen. 
In general, on the one hand, the energy consumed in each iteration is proportional to the increase of the area of the crack surface, that is

$$
\Delta U_{n}=G_{f} \Delta a_{n}
$$

where $G_{f}$ is the fracture energy. On the other hand, the required energy to generate new cracks equal to the area under the softening curve in a interval of length $\Delta w$ implies that

$$
\Delta W_{n}=A \sigma_{n} \Delta w
$$

Thus, form Eqs.(2.10) and (2.11), there is

$$
G_{f} \frac{\Delta a_{n}}{A}=\sigma_{n} \Delta w
$$

The relationship between the stress and the elongation in each iteration can be obtained from Eqs. (2.5), (2.8), (2.9) and (2.12), as

$$
\begin{aligned}
\sigma_{n} & =\frac{G_{f} \Delta a_{n}}{A \Delta w}=\frac{G_{f} A_{p} n_{c} p^{2 m-2}\left(2 q-p^{2}\right) 2^{m} q p^{2 m}\left(p^{2}-q\right)^{n-m}}{A_{1} w_{c} p^{2 n}\left(p^{2}+q\right)\left[p^{2 m}-2^{m}\left(p^{2}-q\right)^{m}\right]} \\
& -\frac{G_{f} A_{p} n_{c} p^{2 m-2}\left(2 q-p^{2}\right) q\left(p^{2}-q\right)^{n}}{A_{1} w_{c} p^{2 n}\left(p^{2}+q\right)\left[p^{2 m}-2^{m}\left(p^{2}-q\right)^{m}\right]} \quad 1 \leqslant n \leqslant n_{c}
\end{aligned}
$$

It should be noticed that the values of both the stress and the elongation in Eq. (2.13) are discrete, starting from $n=1$. In order to obtain a continuous stress-elongation curve in the interval of $\left[0, w_{c}\right]$, the Khezrzadeh and Mofid modification (Khezrzadeh and Mofid, 2006) is used, which is described briefly below. Firstly, it is assumed that the value of the softening function in $w=0$ is equal to the tensile strength of the specimen, i.e., $\sigma(0)=f_{t}$, and that the stress-elongation curve is linear in the interval of $[0, \Delta w]$. Next, an energy modification factor $\mu$ is then introduced to make sure that the area under the softening curve is equal to $G_{f}$, namely,

$$
(1-\mu) G_{f}=\left[f_{t}+\sigma\left(\frac{\Delta w}{2}\right)\right] \frac{\Delta w}{2}
$$

Thus one has

$$
\begin{aligned}
\sigma= & \frac{\mu A_{p} G_{f} n_{c} p^{2 m-2}\left(2 q-p^{2}\right) 2^{m} q p^{2 m}\left(p^{2}-q\right)^{\frac{n_{c}}{w_{c}} w-m}}{A_{1} w_{c} p^{2 \frac{n_{c}}{w_{c}} w}\left(p^{2}+q\right)\left[p^{2 m}-2^{m}\left(p^{2}-q\right)^{m}\right]} \\
& -\frac{\mu A_{p} G_{f} n_{c} p^{2 m-2}\left(2 q-p^{2}\right) q\left(p^{2}-q\right)^{\frac{n_{c}}{w_{c}} w}}{A_{1} w_{c} p^{2 \frac{n_{c}}{w_{c}} w}\left(p^{2}+q\right)\left[p^{2 m}-2^{m}\left(p^{2}-q\right)^{m}\right]} \quad \frac{w_{c}}{n_{c}} \leqslant w \leqslant w_{c}
\end{aligned}
$$

For convenience, we assume that $q=1$ in the ubiquitiformal softening model, then Eq. (2.15) can be rewritten as

$$
\begin{aligned}
\sigma= & \frac{\mu A_{p} G_{f} n_{c} p^{2 m-2}\left(2-p^{2}\right) 2^{m} p^{2 m}\left(p^{2}-1\right)^{\frac{n_{c}}{w_{c}} w-m}}{A_{1} w_{c} p^{2 \frac{n_{c}}{w_{c}} w}\left(p^{2}+1\right)\left[p^{2 m}-2^{m}\left(p^{2}-1\right)^{m}\right]} \\
& -\frac{\mu A_{p} G_{f} n_{c} p^{2 m-2}\left(2-p^{2}\right)\left(p^{2}-1\right)^{\frac{n_{c}}{w_{c}} w}}{A_{1} w_{c} p^{2 \frac{n_{c}}{w_{c}} w}\left(p^{2}+1\right)\left[p^{2 m}-2^{m}\left(p^{2}-1\right)^{m}\right]} \quad \frac{w_{c}}{n_{c}} \leqslant w \leqslant w_{c}
\end{aligned}
$$

For $w=\Delta w$, we have

$$
\begin{gathered}
\sigma(\Delta w)=\frac{\mu A_{p} G_{f} n_{c} p^{2 m-4}\left(2-p^{2}\right) 2^{m} p^{2 m}\left(p^{2}-1\right)^{1-m}}{A_{1} w_{c}\left(p^{2}+1\right)\left[p^{2 m}-2^{m}\left(p^{2}-1\right)^{m}\right]} \\
-\frac{\mu A_{p} G_{f} n_{c} p^{2 m-4}\left(2-p^{2}\right)\left(p^{2}-1\right)}{A_{1} w_{c}\left(p^{2}+1\right)\left[p^{2 m}-2^{m}\left(p^{2}-1\right)^{m}\right]}
\end{gathered}
$$


We can obtain the slope of the softening curve in the interval $[0, \Delta w]$

$$
\begin{aligned}
C= & \frac{\sigma(\Delta w)-f_{t}}{\Delta w}=\frac{\mu A_{p} G_{f} n_{c}^{2} p^{2 m-4}\left(2-p^{2}\right) 2^{m} p^{2 m}\left(p^{2}-1\right)^{1-m}}{A_{1} w_{c}^{2}\left(p^{2}+1\right)\left[p^{2 m}-2^{m}\left(p^{2}-1\right)^{m}\right]} \\
& -\frac{\mu A_{p} G_{f} n_{c}^{2} p^{2 m-4}\left(2-p^{2}\right)\left(p^{2}-1\right)}{A_{1} w_{c}^{2}\left(p^{2}+1\right)\left[p^{2 m}-2^{m}\left(p^{2}-1\right)^{m}\right]}-\frac{n_{c}}{w_{c}} f_{t}
\end{aligned}
$$

Then we have

$$
\begin{aligned}
\sigma= & \frac{\mu A_{p} G_{f} n_{c}^{2} p^{2 m-4}\left(2-p^{2}\right) 2^{m} p^{2 m}\left(p^{2}-1\right)^{1-m}}{A_{1} w_{c}^{2}\left(p^{2}+1\right)\left[p^{2 m}-2^{m}\left(p^{2}-1\right)^{m}\right]} w \\
& -\frac{\mu A_{p} G_{f} n_{c}^{2} p^{2 m-4}\left(2-p^{2}\right)\left(p^{2}-1\right)}{A_{1} w_{c}^{2}\left(p^{2}+1\right)\left[p^{2 m}-2^{m}\left(p^{2}-1\right)^{m}\right]} w-\frac{n_{c}}{w_{c}} f_{t} w+f_{t} \quad 0 \leqslant w \leqslant \frac{w_{c}}{n_{c}}
\end{aligned}
$$

From Eq. (2.14) and Eq. (2.19) one can obtain the energy modification factor $\mu$ as

$$
\begin{aligned}
\mu= & 1-\frac{A_{p} p^{2 m-4}\left(2-p^{2}\right)\left[2^{m} p^{2 m}\left(p^{2}-1\right)^{1-m}-\left(p^{2}-1\right)\right]}{4 A_{1}\left(p^{2}+1\right)\left[p^{2 m}-2^{m}\left(p^{2}-1\right)^{m}\right]}-\frac{3 f_{t} w_{c}}{4 G_{f} n_{c}}=\frac{4 G_{f} n_{c}-3 f_{t} w_{c}}{4 G_{f} n_{c}} \\
& \cdot \frac{4 A_{1}\left(p^{2}+1\right)\left[p^{2 m}-2^{m}\left(p^{2}-1\right)^{m}\right]}{A_{p} p^{2 m-4}\left(2-p^{2}\right)\left[2^{m} p^{2 m}\left(p^{2}-1\right)^{1-m}-\left(p^{2}-1\right)\right]+4 A_{1}\left(p^{2}+1\right)\left[p^{2 m}-2^{m}\left(p^{2}-1\right)^{m}\right]}
\end{aligned}
$$

The ubiquitiformal softening curve of concrete is then

$$
\sigma= \begin{cases}\frac{\mu A_{p} G_{f} n_{c} p^{2 m-2}\left(2-p^{2}\right)\left[2^{m} p^{2 m}\left(p^{2}-1\right)^{-m}-1\right]}{A_{1} w_{c}\left(p^{2}+1\right)\left[p^{2 m}-2^{m}\left(p^{2}-1\right)^{m}\right]} & \left.\frac{1}{p^{2}}\right)^{\frac{n_{c}}{w_{c}}} \leqslant w \leqslant w_{c} \\ \left(\frac{\mu A_{p} G_{f} n_{c}^{2} p^{2 m-4}\left(2-p^{2}\right)\left[2^{m} p^{2 m}\left(p^{2}-1\right)^{1-m}-\left(p^{2}-1\right)\right]}{A_{1} w_{c}^{2}\left(p^{2}+1\right)\left[p^{2 m}-2^{m}\left(p^{2}-1\right)^{m}\right]}-\frac{n_{c}}{w_{c}} f\right) w+f_{t} & 0 \leqslant w \leqslant \frac{w_{c}}{n_{c}}\end{cases}
$$

In the ubiquitiformal softening model, the iteration number is calculated by the relation

$$
\left(\frac{1}{p}\right)^{N}=\frac{\delta_{\min }}{l}
$$

where $\delta_{\min }$ and $l$ are the minimum and maximum scales of the concrete respectively, which are related to the micro and macro structure of the concrete. However, the iteration number calculated from Eq. (2.22) is always not an integer, whereas the iteration number of the generalized ubiquitiformal Cantor set should be an integer. Thus we assume that $n=[N]$ in this paper, where the square brackets represents the maximum integer no larger than the argument.

\section{Numerical results of full grade concrete}

To confirm the availability of the ubiquitiformal softening model, the model is used to calculate the softening curve of a full grade concrete specimen made of crushed coarse aggregate, and the numerical results are compared with the experimental result (Deng et al., 2005). In the experiment, the uniaxial tension-compression behavior of the full grade concrete specimens made of crushed coarse aggregate was studied on an INSTRON8506 material testing machine under constant-displacement loading, with the maximum load of $3000 \mathrm{kN}$. Four displacement extensometers were set around the test specimen, and the data collection and the loading control were completed by using a computer. The composition of the concrete and the experimental data are listed in Tables 1 and 2, respectively. 
Table 1. Concrete mix of the concrete $\left[\mathrm{kg} / \mathrm{m}^{3}\right]$

\begin{tabular}{|c|c|c|c|c|c|c|c|c|c|}
\hline \multirow{2}{*}{ Water } & \multirow{2}{*}{ Cement } & \multirow{2}{*}{ Ash } & Artificial & \multicolumn{4}{|c|}{ Artificial stone [mm] } & \multicolumn{2}{c|}{ Superplasticizerits } \\
\cline { 5 - 9 } & & & sand & $5-20$ & $20-40$ & $40-80$ & $80-150$ & JGB & DH9 \\
\hline \hline 87 & 131 & 44 & 585 & 328 & 328 & 492 & 492 & 10.5 & 1.23 \\
\hline
\end{tabular}

Table 2. Experimental data of the concrete specimen

\begin{tabular}{|c|c|c|c|c|}
\hline $\begin{array}{c}\text { Curing period } \\
\text { [day }]\end{array}$ & $\begin{array}{c}\text { Tensile strength } \\
f_{t}[\mathrm{MPa}]\end{array}$ & $\begin{array}{c}\text { Elastic modulus } \\
E_{t}[\mathrm{GPa}]\end{array}$ & $\begin{array}{c}\text { Critical elongation } \\
w_{c}[\mathrm{~mm}]\end{array}$ & $\begin{array}{c}\text { Fracture energy } \\
G_{f}[\mathrm{~N} / \mathrm{m}]\end{array}$ \\
\hline \hline 110 & 1.908 & 40.0 & 1.390 & 497.220 \\
\hline 55 & 1.508 & 37.0 & 1.355 & 448.401 \\
\hline 46 & 1.310 & 35.0 & 1.199 & 422.878 \\
\hline 16 & 1.180 & 31.1 & 1.680 & 445.738 \\
\hline 15 & 1.044 & 28.9 & 1.289 & 369.463 \\
\hline 11 & 0.804 & 22.0 & 1.193 & 273.233 \\
\hline
\end{tabular}

In the ubiquitiformal softening model, the parameter is: $p=2.07$, which is the same as in Khezrzadeh and Mofid (2006), and the adaptive result for $m$ is $m=2$.

For a certain concrete, the parameters $p, m, A_{p}$ and $n_{c}$ in the softening model are determined, and the material parameters $G_{f}, w_{c}$ and $f_{t}$ are also known. Thus the parameter $A_{1}$ and $\mu$ can be regarded as constants. Therefore, for convenience, we rewrite Eq. (2.21) as

$$
\sigma= \begin{cases}C_{1} C_{2}^{w} & \frac{w_{c}}{n_{c}} \leqslant w \leqslant w_{c} \\ f_{t}-C_{3} w & 0 \leqslant w \leqslant \frac{w_{c}}{n_{c}}\end{cases}
$$

where $C_{1}, C_{2}$ and $C_{3}$ are constant. The values of these parameters for concrete specimens with different curing periods as well as the experimental data are all listed in Table 3.

Table 3. Parameters of the ubiquitiformal softening model for concrete specimen

\begin{tabular}{|c|c|c|c|c|c|c|c|}
\hline $\begin{array}{c}\text { Curing } \\
\text { period [day] }\end{array}$ & $\begin{array}{c}\text { Tensile strength } \\
f_{t}[\mathrm{MPa}]\end{array}$ & $\begin{array}{c}\text { Crit. elongation } \\
w_{c}[\mathrm{~mm}]\end{array}$ & $\begin{array}{c}\text { Iteration } \\
\text { number } n_{c}\end{array}$ & $\begin{array}{c}\delta_{\min } \\
{[\mu \mathrm{m}]}\end{array}$ & $C_{1}$ & $C_{2}$ & $C_{3}$ \\
\hline \hline 110 & 1.908 & 1.390 & 12 & 24 & 1.145 & 0.1008 & 8.894 \\
\hline 55 & 1.508 & 1.355 & 11 & 50 & 1.0047 & 0.1156 & 5.9895 \\
\hline 46 & 1.310 & 1.199 & 10 & 104 & 1.0188 & 0.1090 & 4.4188 \\
\hline 16 & 1.180 & 1.680 & 10 & 104 & 0.7080 & 0.2055 & 3.7931 \\
\hline 15 & 1.044 & 1.289 & 9 & 215 & 0.7194 & 0.1563 & 3.4388 \\
\hline 11 & 0.804 & 1.193 & 8 & 445 & 0.4916 & 0.1682 & 2.8644 \\
\hline
\end{tabular}

The comparison between the softening curves calculated by using the ubiquitiformal model and the experimental results are shown in Fig. 2. It can be seen that the ubiquitiformal softening model is in good agreement with the experimental data. It should be pointed out that the difference of the stress between the softening curve in the interval $[0, \Delta w]$ increases with the tensile strength of the specimen, except for the specimen with a curing period of 16 days. However, it can also be seen that the experimental data for this specimen, especially the critical elongation, is abnormal. The difference of the softening curve of this specimen is caused mainly by abnormality of the experimental data.

As has been mentioned by $\mathrm{Ou}$ et al. (2014), the lower bound to the scale invariance $\delta_{\text {min }}$, namely, the minimum scale of concrete, is a crucial parameter for a ubiquitiform, and it can be 
(a)

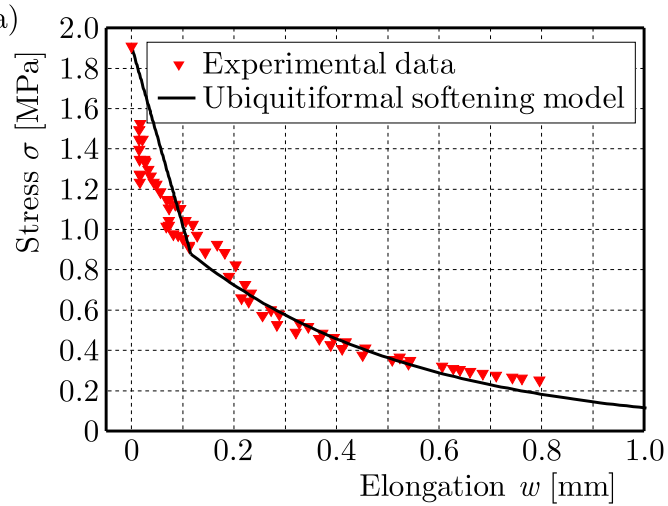

(c)

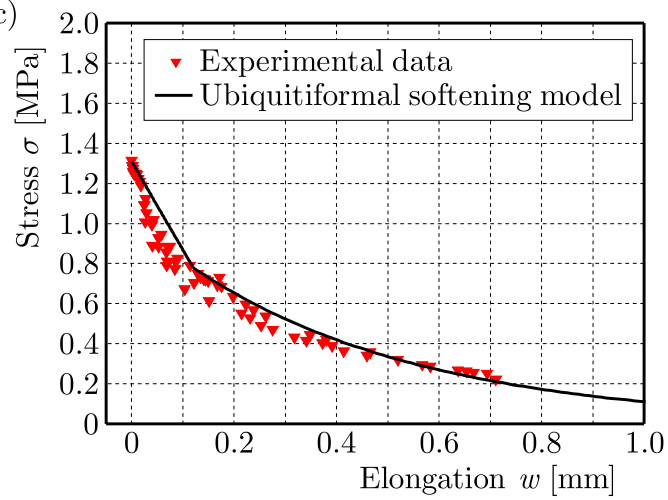

(e)

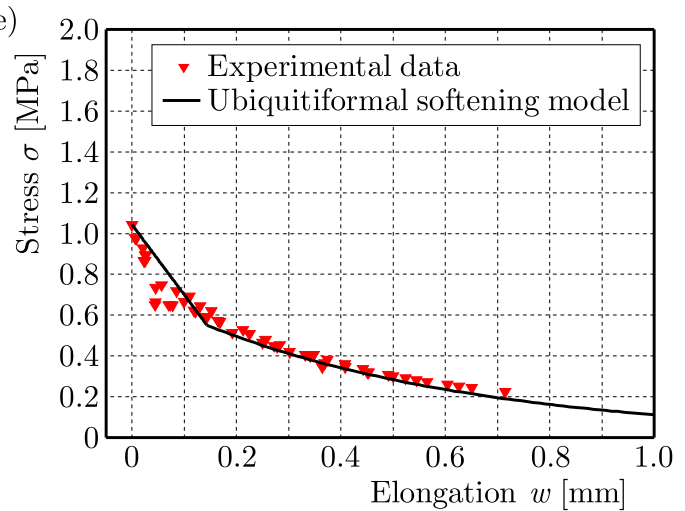

(b)

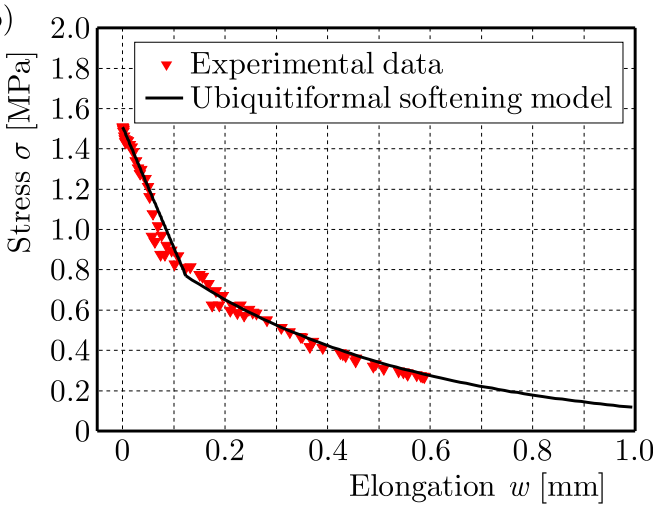

(d)

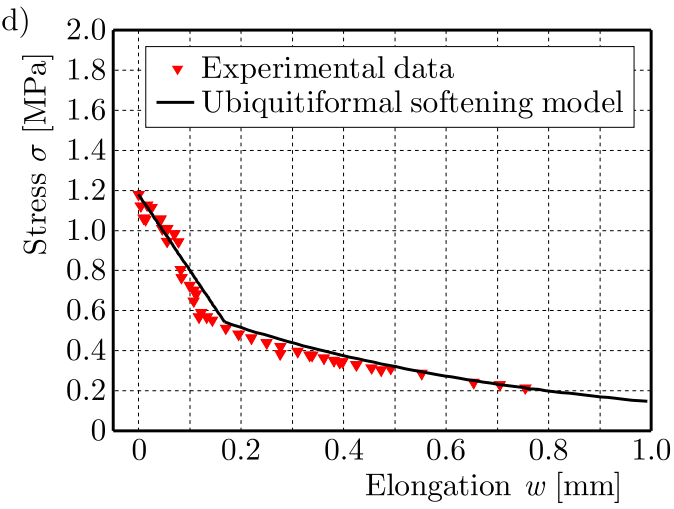

(f)

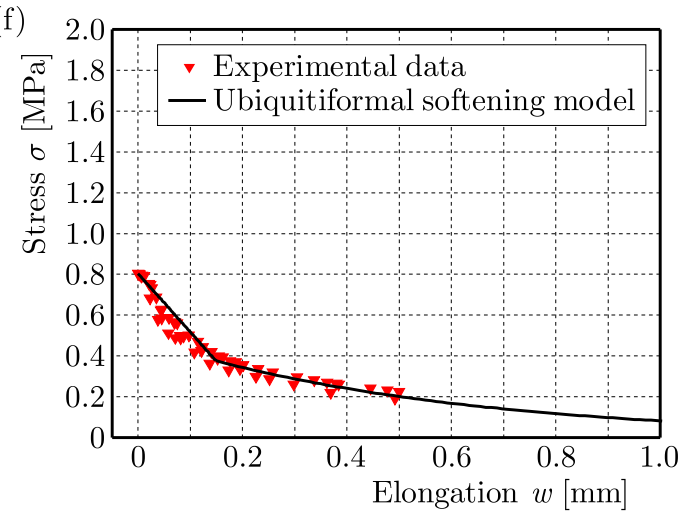

Fig. 2. The ubiquitiformal softening curve: (a) specimen of $1.908 \mathrm{MPa}$, (b) specimen of 1.508 MPa, (c) specimen of $1.310 \mathrm{MPa}$, (d) specimen of $1.180 \mathrm{MPa}$, (e) specimen of 1.044 MPa, (f) specimen of $0.804 \mathrm{MPa}$

seen that this crucial parameter is related with the tensile strength of the concrete specimen with different curing periods. The lower bound to the scale invariance $\delta_{\min }$ for the specimen with different tensile strength is shown in Fig. 3. By fitting the data with a power function, the relation between the lower bound to the scale invariance $\delta_{\min }$ and the tensile strength can be obtained as

$$
\delta_{\text {min }}=221.28 \cdot f_{t}^{-3.24}
$$

where the units of $\delta_{\min }$ and $f_{t}$ are $\mu \mathrm{m}$ and $\mathrm{MPa}$, respectively. This relationship provides a reasonable approach to determine the lower bound to the scale invariance of concrete. Furthermore, by analysing the influencing factors of the concrete tensile strength, the approach to determine the lower bound to the scale invariance of concrete by other physical parameters may be obtained. 


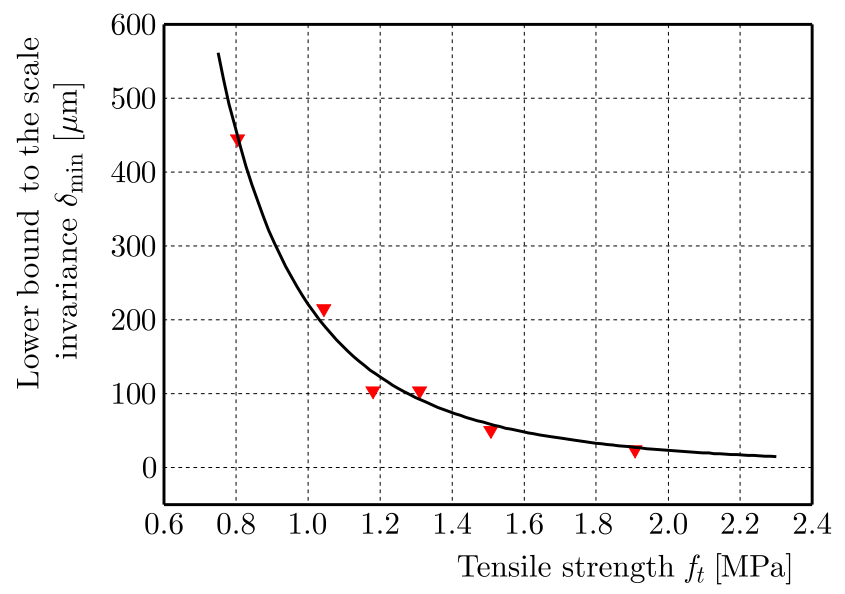

Fig. 3. The relation between the lower bound to the scale invariance $\delta_{\min }$ and tensile strength of concrete

It should be mentioned that, although such an ubiquitiformal softening model for concrete seems to be similar to the fractal one (Khezrzadeh and Mofid, 2006), it has more definite physical meanings. The relation between the lower bound to the scale invariance and tensile strength of concrete is obtained numerically, which may provide a reasonable approach to determine the lower bound to the scale invariance of concrete.

\section{Conclusion}

A stereological type of ubiquitiformal softening model that can well describe the softening behavior of concrete under quasi-static tensile loadings is proposed in this paper. Both the damage evaluation process of fracture cross-sections and their distribution along the specimens axis are considered. Moreover, by fitting the experimental data, a relation between the lower bound to the scale invariance and the tensile strength of concrete is obtained, which provides a reasonable approach to determine the lower bound to the scale invariance of concrete.

\section{Acknowledgements}

This work were supported by The National Natural Science Foundation of China under Grant 11772056 and also The National Science Foundation of China under Grant 11521062.

\section{References}

1. Barenblatt G.I., 1959, The formation of equilibrium cracks during brittle fracture: General ideas and hypotheses, axially symmetric cracks, Journal of Applied Mathematics and Mechanics, 23, $622-636$

2. Barenblatt G.I., 1962, The mathematical theory of equilibrium cracks in brittle fracture, $A d$ vances in Applied Mechanics, 7, 55-129

3. BAŽAnt Z.P., Он B.H., 1983, Crack band theory for fracture of concrete, Matériaux et Construction, 16, 155-177

4. Borodich F.M., 1997, Some fractal models of fracture, Journal of the Mechanics and Physics of Solids, 45, 239-259

5. Carpinteri A., Chiaia B., Cornetti P., 2002, A scale-invariant cohesive crack model for quasibrittle materials, Engineering Fracture Mechanics, 69, 207-217 
6. Charkaluk E., Bigerelle M., Iost A., 1998, Fractals and fracture, Engineering Fracture Mechanics, 61, 119-139

7. Deng Z.C., Li Q.B., Fu H., 2005, Mechanical properties of tension and compression about artificial full-graded aggregate concrete dam (in Chinese), Journal of Hydraulic Engineering, 36, $214-218$

8. Gopalaratnam V.S., Shah S.P., 1985, Softening response of plain concrete in direct tension, Journal Proceedings (American Concrete Institute), 82, 310-323

9. Hillerborg A., Modeer M., Petersson P.E., 1976, Analysis of crack formation and crack growth in concrete by means of fracture mechanics and finite elements, Cement and Concrete Research, 6, 773-782

10. Karihaloo B.L., 1995, Fracture Mechanics and Structural Concrete, Longman Scientific \& Technical, Harlow

11. Khezrzadeh H., Mofid M., 2006, Tensile fracture behavior of heterogeneous materials based on fractal geometry, Theoretical and Applied Fracture Mechanics, 46, 46-56

12. Mandelbrot B.B., 1982, The Fractal Geometry of Nature, Freeman, New York

13. Mandelbrot B.B., Passoja D.E., Paullay A.J., 1984, Fractal character of fracture surfaces of metals, Nature, 308, 721-722

14. Ou Z.C., Li G.Y., Duan Z.P., Huang F.L., 2014, Ubiquitiform in applied mechanics, Journal of Theoretical and Applied Mechanics, 52, 37-46

15. Petersson P.E., 1981, Crack growth and development of fracture zone in plain concrete and similar materials, Division of Building Materials, Lund Institute of Technology

16. Reinhardt H.W., Cornelissen H.A.W., Hordijk D.A., 1986, Tensile tests and failure analysis of concrete, Journal of Structural Engineering, 112, 2462-2477

17. Saouma V.E., Barton C.C., 1994, Fractals, fractures, and size effects in concrete, Journal of Engineering Mechanics, 120, 835-854

18. Stroeven P., 1973, Some aspects of the micro-mechanics of concrete, Ph.D. Thesis, Delft University of Technology, Delft

19. Stroeven P., 2000, A stereological approach to roughness of fracture surfaces and tortuosity of transport paths in concrete, Cement and Concrete Composites, 22, 331-341 